\title{
Clinical validation of 13-gene DNA methylation analysis in oral brushing samples for detection of oral carcinoma: An Italian multicenter study
}

\author{
Davide B. Gissi PhD, DDS ${ }^{1}$ (i) ～Luca Morandi PhD, BSc ${ }^{2} \quad$ Giuseppe Colella MD $^{3}$ | \\ Roberto De Luca $\mathrm{MD}^{3}$ (D) | Giuseppina Campisi $\mathrm{SSD}^{4}$ | \\ Rodolfo Mauceri PhD, DDS ${ }^{4}$ | Umberto Romeo DDS ${ }^{5}$ | \\ Gianluca Tenore PhD, DDS ${ }^{5}$ | Michele D. Mignogna MD $^{6}$ | \\ Daniela Adamo DDS ${ }^{6} \quad$ Giacomo Oteri MD, DDS ${ }^{7} \quad$ Antonia Marcianò DDS ${ }^{7}$ | \\ Paolo Vescovi DDS ${ }^{8} \quad$ Marco Meleti PhD, DDS $^{8} \quad$ Rosario Serpico MD, DDS ${ }^{3}$ | \\ Dario Di Stasio PhD, DDS ${ }^{3} \quad \mid$ Monica Pentenero DDS ${ }^{9} \quad \mid$ Andrea Santarelli DDS ${ }^{10}$ | \\ Alberto Bianchi MD, DDS ${ }^{11}$ | Salvatore Crimi MD ${ }^{11}$ | \\ Claudio Marchetti MD, DDS ${ }^{12}$ | Achille Tarsitano MD $^{12}$ | \\ Maria Pia Foschini MD M $^{13}$ Lucio Montebugnoli MD, DDS ${ }^{1}$ \\ ${ }^{1}$ Department of Biomedical and Neuromotor Sciences, Section of Oral Sciences, University of Bologna, Bologna, Italy \\ ${ }^{2}$ Functional MR Unit, IRCCS Istituto delle Scienze Neurologiche di Bologna, Department of Biomedical and Neuromotor Sciences, University of \\ Bologna, Bologna, Italy \\ ${ }^{3}$ Multidisciplinary Department of Medical-Surgical and Dental Specialities, University of Campania “L. Vanvitelli”, Naples, Italy \\ ${ }^{4}$ Department of Surgical, Oncological, and oral Sciences, Sector of Oral Medicine "Valerio Margiotta", University of Palermo, Palermo, Italy \\ ${ }^{5}$ Department of Oral and Maxillofacial Sciences, "Sapienza” University of Rome, Rome, Italy \\ ${ }^{6}$ Oral Medicine Complex Unit, Department of Neurosciences, Reproductive and Odontostomatological Sciences - Head \& Neck Clinical Section, \\ "Federico II" University of Naples, Naples, Italy \\ ${ }^{7}$ Department of Biomedical, Dental Sciences and Morphofunctional Imaging, University of Messina, Messina, Italy \\ ${ }^{8}$ Oral Medicine and Laser Surgery Unit, Department of Medicine and Surgery, University of Parma, Parma, Italy \\ ${ }^{9}$ Oral Medicine and Oral Oncology Unit, Department of Oncology, University of Turin, Turin, Italy \\ ${ }^{10}$ Department of Clinical Sciences and Stomatology, Marche Polytechnic University, Ancona, Italy \\ ${ }^{11}$ Section of Maxillo-Facial Surgery, Department of General Surgery, Policlinico - San Marco, University of Catania, Catania, Italy \\ ${ }^{12}$ Section of Maxillo-Facial Surgery at Policlinico S. Orsola-Malpighi, Department of Biomedical and Neuromotor Sciences, University of Bologna, \\ Bologna, Italy \\ ${ }^{13}$ Section of Anatomic Pathology at Bellaria Hospital, Department of Biomedical and Neuromotor Sciences, University of Bologna, Bologna, Italy
}

\section{Correspondence}

Davide B. Gissi, Department of

Biomedical and Neuromotor Sciences,

Section of Oral Sciences, University of

Bologna, Via San Vitale 59, 40159

Bologna, Italy.

Email: davide.gissi@unibo.it

Funding information

University of Bologna

\section{Abstract}

Background: The aim of this Italian multicenter study was to evaluate the diagnostic performance of a minimally invasive method for the detection of oral squamous cell carcinoma (OSCC) based on 13-gene DNA methylation analysis in oral brushing samples.

Methods: Oral brushing specimens were collected in 11 oral medicine centers across Italy. Twenty brushing specimens were collected by each center, 10 from 
patients with OSCC, and 10 from healthy volunteers. DNA methylation analysis was performed in blindness, and each sample was determined as positive or negative based on a predefined cutoff value.

Results: DNA amplification failed in 4 of $220(1.8 \%)$ samples. Of the specimens derived from patients with OSCC, 93.6\% (103/110) were detected as positive, and $84.9 \%$ (90/106) of the samples from healthy volunteers were negative. Conclusion: These data confirmed the diagnostic performance of our novel procedure in a large cohort of brushing specimens collected from 11 different centers and analyzed in blindness.

\section{KEYWOR D S}

algorithm, bisulfite sequencing, diagnostic test, oral brushing, oral squamous cell carcinoma, quantitative DNA methylation analysis

\section{1 | INTRODUCTION}

Oral squamous cell carcinoma (OSCC) poses a critical challenge to public health. Despite the advent of new surgery, radiotherapy, and chemotherapy treatment modalities for the management of patients with OSCC, the 5-year mortality rate remains approximately $60 \%{ }^{1}$ The prognosis for OSCC of the tongue, the most common type of OSCC, also remains very poor. ${ }^{2}$ Furthermore, the development of a second primary OSCC after surgery is not uncommon, with frequency ranging between $17 \%$ and $30 \%{ }^{3}$ This rate is higher than any other type of tumor and is a leading cause of cancer-related death. ${ }^{4}$ Although the oral cavity is easily accessible for examination, only $29 \%$ of oral cancers are detected at an early stage and before metastasis to other sites. ${ }^{5}$ This is largely due to the marked biological propensity for local invasion and the high incidence of cervical lymph node metastasis at initial diagnosis (40\%). ${ }^{6}$

In current clinical practice, oral cancer screening is performed solely by oral visual examination, including inspection and palpation, by general physicians or dentists. In cases of suspicious neoplastic or preneoplastic lesions, patients are referred to oral medicine specialists or oral/maxillofacial surgeons for a detailed visual oral examination and further diagnostic biopsy. However, early detection might be hindered by practitioners' clinical experience and the subtle nature of the lesions, which may be undetectable by oral examination alone. Thomson et al. reported the presence of premalignant lesions and micro-invasive carcinomas in clinically normalappearing oral mucosa. ${ }^{7}$ The development of a noninvasive or minimally invasive diagnostic tool for OSCC screening and longitudinal monitoring of patients at high risk of OSCC development, such as patients with oral potentially malignant disorders (OPMDs) or patients surgically treated for OSCC, may provide early OSCC diagnosis and improve the survival of patients with OSCC.

Currently, no method allowing for screening, early detection, or monitoring of OSCC in high-risk groups is commercially available. ${ }^{8}$ In the past few decades, several noninvasive diagnostic tools have been proposed, including toluidine blue staining, autofluorescence, chemiluminescence, exfoliative cytology, and optical diagnostic method, such as Raman spectroscopy, elastic scattering, diffuse reflectance, narrow-band imaging, and fluorescence visualization. ${ }^{9,10}$ However, these methods have several limitations, and there is no evidence indicating that their use could reduce cancer mortality. ${ }^{11}$ Collection of samples from oral brushing, mouth rinsing, or saliva for analysis of OSCC biomarkers has been proposed as an alternative, noninvasive, or minimally invasive screening strategy. In recent years, a large number of OSCC biomarkers have been identified including DNA image cytometry analyzed from quantitative cytology, mRNAs, microRNAs, proteins, and methylation patterns. For example, since late 1992, more than 120 biomarkers have been proposed as diagnostic tools of oral cancer, based on more than 100 studies. $^{12}$ Nevertheless, only a few of them have been validated in clinical samples, and none has been implemented in clinical practice thus far.

We have recently developed an oral-brushing-based minimally invasive method to detect oral carcinomas at an early stage by measuring the DNA methylation levels of a panel of 13 genes. $^{13}$ In a single-center study, the methylation profiles of ZAP70, ITGA4, KIF1A, PARP15, EPHX3, NTM, LRRTM1, FLI1, MIR193, LINC00599, MIR296, TERT, and GP1BB were analyzed, and a score weighting of the best $\mathrm{CpGs}$ from all the 13 genes was calculated. In total, $96.6 \%(n=28)$ of OSCC and $100 \%$ $(n=6)$ of high-grade squamous intraepithelial lesion 
(HGSIL) specimens exceeded the threshold value, whereas none of the healthy donor samples $(n=65)$ exceeded the threshold. ${ }^{13}$ The clinical relevance of the method in oral oncology has been confirmed in patients surgically treated for OSCC; the 13-gene DNA methylation analysis of samples taken from the regenerative area after OSCC resection could identify patients at risk of recurrence during follow-up. ${ }^{14}$

However, the clinical usefulness of minimally invasive procedures for screening and surveillance in oral oncology should be comprehensively explored in large cohort studies and validated in different clinical samples from different institutions. The aim of this multicenter study was to assess the sensitivity, specificity, and reproducibility of the 13-gene DNA methylation analysis in brushing samples from patients with OSCC and healthy volunteers from 11 different Italian academic oral medicine units.

\section{2 | MATERIALS AND METHODS}

\section{1 | Study design}

This prospective study was conducted from March 2018 to December 2019 using patient samples from 11 oral medicine units across Italy. The study was coordinated by the Department of Biomedical and Neuromotor Sciences, Section of Oral Sciences, University of Bologna. All clinical investigations were conducted according to the principles of the Declaration of Helsinki. The study protocol was approved by the ethics committee of Bologna (study number: 520/2018/Sper/AOUBo; protocol number: OB-200) and a written informed consent was obtained from all patients. All information regarding the human material used in this study was managed using anonymous numerical codes.

\section{2 | Study population and brushing sampling}

Each oral medicine unit collected 20 brushing specimens, 10 from patients with OSCC, and 10 from healthy volunteers. Oral brushing sample collection was performed by clinicians participating in the study using a previously described method. ${ }^{13,15,16}$ Briefly, all samples were collected using the same cytobrush (NHMP Co., Ltd., PRC EC REP, Shanghai International Trading Corporation, Hamburg, Germany). Exfoliated cells from oral mucosa were collected after brushing. Each cytobrush sample was placed in a 2-ml tube containing $500 \mu \mathrm{l}$ of DNA/RNA-Shield (Zymo Research, Irvine, CA) for nucleic acid preservation. Brushing specimens were collected by one operator from each participating center. In patients with suspected oral neoplastic lesions, the surface of the lesion was gently brushed with rotation and translation movements. In these patients, oral brushing was performed before incisional biopsy, and samples were used in the study only after histological confirmation of OSCC. Clinical information obtained from each patient with OSCC included age, sex, smoking habits, clinical appearance, tumor location, and clinical $\mathrm{T}$ (cT) classification. cT classification was based on the oral examination, imaging data (head and neck CT-scan and/or MRI), and biopsies of affected areas. Control brushing specimens were collected from healthy volunteers who presented at each University center during the study period only for dental care; the healthy volunteers were matched for age, sex, and smoking habits. Samples from healthy volunteers who had lesions of any type in the oral cavity (malignant, potentially malignant, infective, reactive, or benign) were excluded from the study. In healthy volunteers the surface of different sites of the oral mucosa (cheeks, marginal, and dorsal tongue) was gently brushed with rotation and translational movements. Samples from each oral medicine unit were submitted to the coordinating center as masked samples. All brushing samples were analyzed by the same geneticist (L.M.), who had no direct access to the codes used for masking the specimens and who was unaware of the clinical and provenance information.

\section{3 | DNA methylation analysis}

DNA methylation analysis was performed as previously described by Morandi et al. ${ }^{13}$ Briefly, DNA from exfoliated cells was purified using The MasterPure Complete DNA Purification Kit (MC85200; Lucigen, Middleton, WI) and treated with sodium bisulfite using the EZ DNA Methylation-Lightning Kit (D5031; ZymoResearch, Irvine, CA) according to the manufacturer's instructions. Quantitative DNA methylation analysis was performed by next-generation sequencing for the following genes: ZAP70, ITGA4, KIF1A, PARP15, EPHX3, NTM, LRRTM1, FLI1, MIR193, LINC00599, MIR296, TERT, and GP1BB. Libraries were prepared using the Nextera Index Kit by a locus-specific bisulfite amplicon approach ${ }^{17}$ and loaded onto MiSEQ (15027617; Illumina, San Diego, CA). FASTQ output files were processed for quality control (>Q30) and converted into FASTA format in a Galaxy Project environment. ${ }^{18}$ The methylation ratio of each CpG was calculated in parallel by different tools: BSPAT (http://cbc.case.edu/BSPAT/index.jsp), ${ }^{19}$ BWAmeth in a Galaxy Project environment (Europe) followed by the 
MethylDackel tool (https://github.com/dpryan79/ MethylDackel), EPIC-TABSAT, ${ }^{20}$ and Kismeth. ${ }^{21}$ The web tool EasyROC ${ }^{22}$ was used to calculate the area under the curve (AUC) for each CpG of the 13 genes and to create the heatmap, and ClustVis ${ }^{23}$ was used for the principal component analysis (PCA).

In our previous study, ${ }^{13}$ the best $\mathrm{CpGs}$ identified by receiver operating characteristics (ROC) analysis were used to generate a choice algorithm based on multiclass linear discriminant analysis. This allowed us to correctly identify OSCC with a threshold of 1.0615547 as the best value for sensitivity and specificity $($ AUC $=0.981)$. Using the same algorithm, in this study, we calculated a specific score for each sample. Values exceeding the threshold of 1.0615547 were considered positive. The study design is summarized in the flowchart shown in Figure 1.

\section{$2.4 \quad$ Statistical analysis}

Each sample was analyzed as either a numeric or a dichotomous variable (positive/negative), according to the score generated from the algorithm and the cutoff value calculated previously. ${ }^{13}$ Kruskal-Wallis analysis

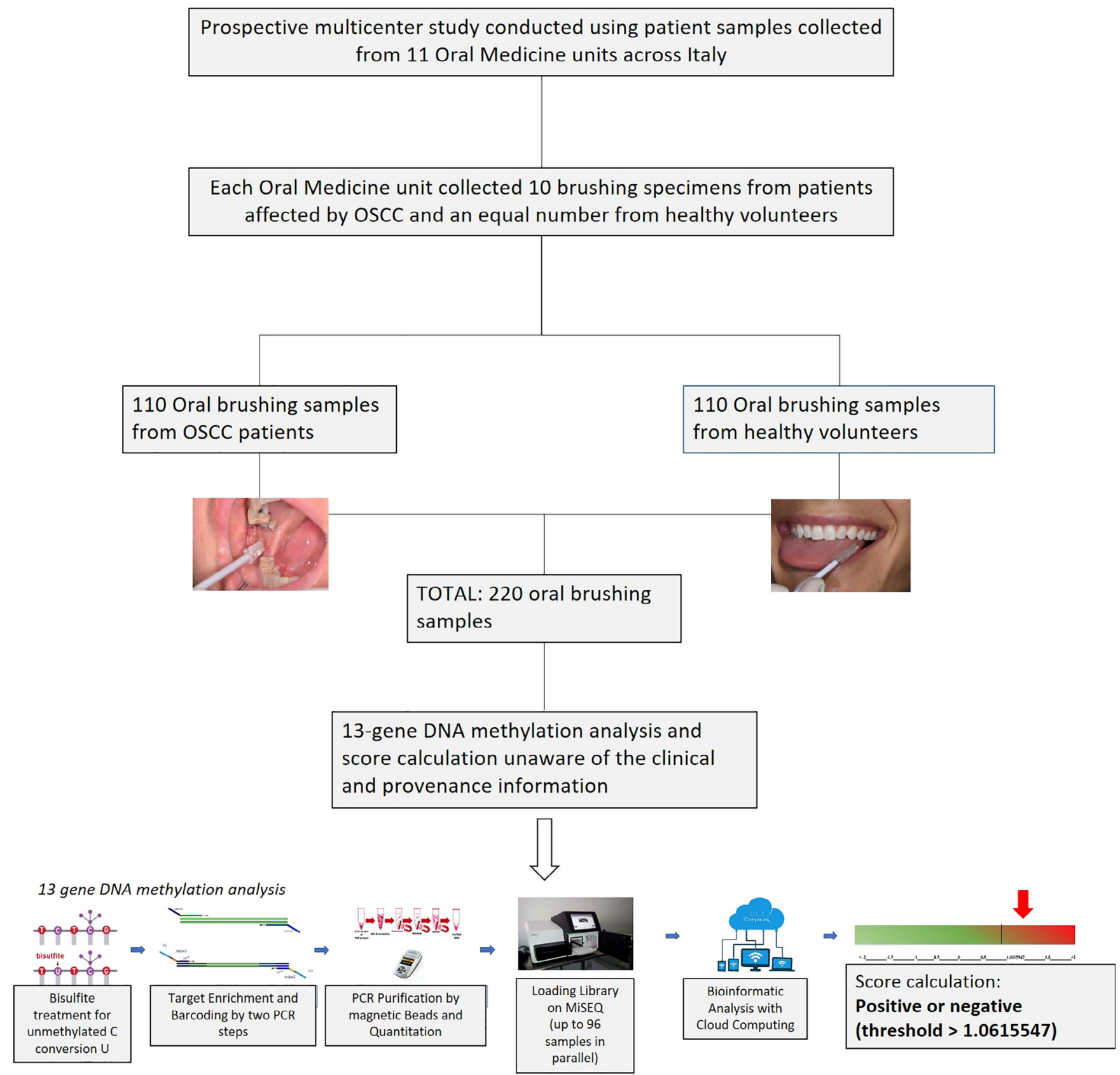

F I G U RE 1 Flowchart summarizing the study design [Color figure can be viewed at wileyonlinelibrary.com] 


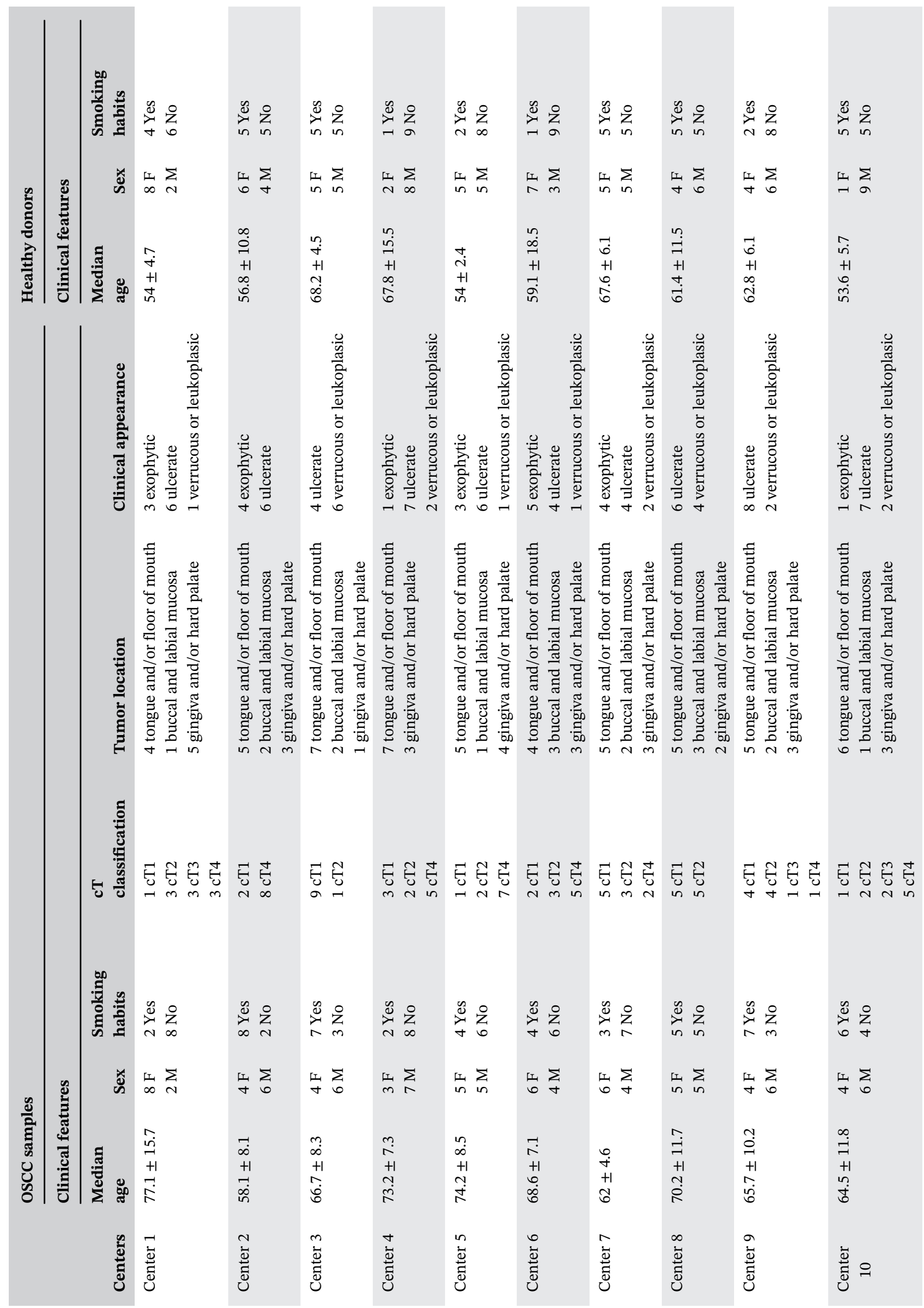




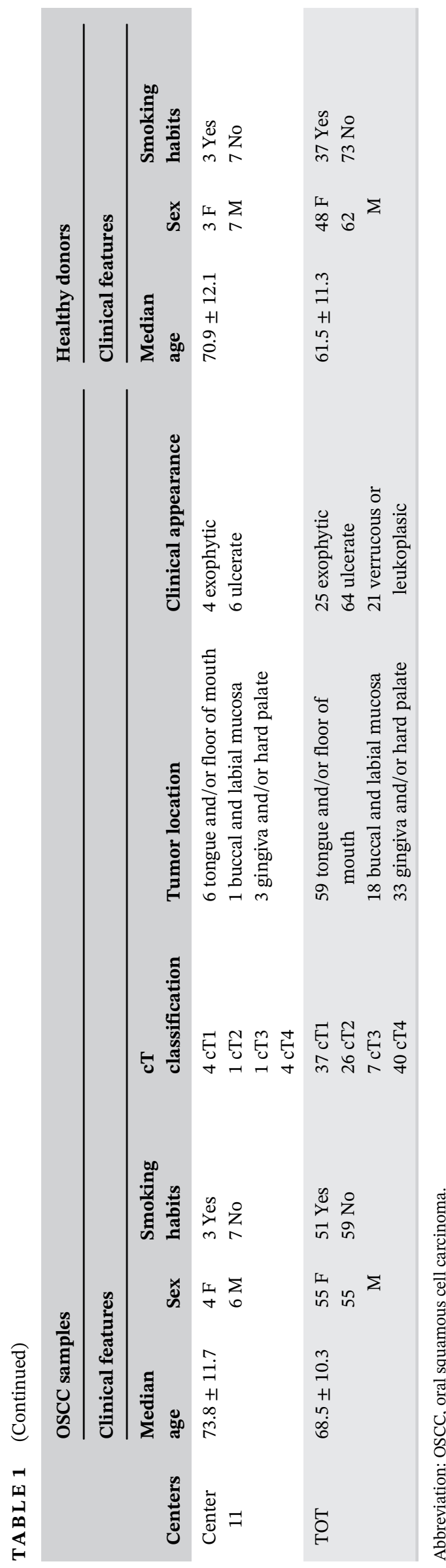

and Fisher's exact test were used to determine significant differences between patients with OSCC and healthy volunteers, as well as to evaluate patient variables influencing the methylation profile in each group (age, sex, smoking habits for both groups and cT classification, clinical appearance, and tumor location only for OSCC group). Kruskal-Wallis analysis was also used to evaluate differences for each $\mathrm{CpG}$ investigated between the groups. $p$-values $<0.05$ were considered statistically significant in all analyses.

Using the generated score and ROC analysis, we evaluated accuracy, sensitivity, and specificity by calculating the AUC. To assess the diagnostic value of the 13-gene DNA methylation analysis for oral cancer detection, we calculated the sensitivity, specificity, positive predictive value (PPV), and negative predictive value (NPV) with the associated $95 \%$ confidence intervals (CIs).

\section{3 | RESULTS}

\section{1 | Study population}

A total of 220 oral brushing specimens were collected and analyzed from 110 patients with OSCC and 110 healthy volunteers. Patients with OSCC included $55(50 \%)$ females and 55 (50\%) males ranging in age from 46 to 97 (median age $68.5 \pm 10.3$ ). Fifty-one $(46.4 \%$ ) patients with OSCC were smokers, whereas the remaining $59(53.6 \%)$ patients were nonsmokers. Fiftynine $(53.6 \%)$ OSCC lesions were located on tongue and/or floor of mouth, 18 (16.4\%) were located on right or left cheek or labial mucosa, and $33(30 \%)$ neoplastic lesions were located on hard palate and/or gingiva. Twenty-five (22.7\%) neoplastic lesions showed a proliferative aspect, 64 (58.2\%) OSCC lesions showed an ulcerative and/or erythroplasic aspect and finally 21 (19.1\%) malignant lesions showed a verrucous and/or a leukoplasic aspect.

Finally, 37 (33.6\%) neoplastic lesions were cT1, $26(23.6 \%)$ were cT2, 7 (6.4\%) were cT3, and 40 (36.4\%) were cT4.

Healthy volunteers included 48 females and 62 males ranging in age from 32 to 85 (median age $61.5 \pm 11.3$ ). Thirty-seven (33.6\%) healthy volunteers were smokers and $73(66.4 \%)$ were nonsmokers. The patient characteristics were summarized in Table 1.

DNA amplification was not possible in 4 of the $220(1.8 \%)$ samples; thus, the final brushing sample collection consisted of 216 brushing specimens, including 110 brushing specimens from patients with OSCC and 106 from healthy volunteers. 
T A B LE 2 Score samples for each oral medicine unit

\begin{tabular}{|c|c|c|c|c|c|c|}
\hline Centers & \multicolumn{3}{|l|}{ OSCC samples } & \multicolumn{3}{|l|}{ Healthy donors } \\
\hline Center 1 & $3.68 \pm 1.9$ & 10 & 0 & $-0.18 \pm 0.9$ & 1 & 9 \\
\hline Center $2^{\mathrm{a}}$ & $1.04 \pm 1.7$ & 6 & 4 & $0.86 \pm 0.3$ & 4 & 5 \\
\hline Center 4 & $2.93 \pm 1.9$ & 9 & 1 & $0.61 \pm 0.7$ & 3 & 7 \\
\hline Center 5 & $2.87 \pm 1.4$ & 10 & 0 & $0.09 \pm 1.3$ & 2 & 8 \\
\hline Center $6^{\mathrm{b}}$ & $3.13 \pm 0.8$ & 10 & 0 & $0.18 \pm 1.1$ & 1 & 7 \\
\hline Center 7 & $3.02 \pm 1.2$ & 10 & 0 & $-0.67 \pm 1.16$ & 1 & 9 \\
\hline Center 11 & $3.07 \pm 1.4$ & 10 & 0 & $-1.16 \pm 0.6$ & 0 & 10 \\
\hline TOT & $2.7 \pm 1.6$ & 103 & 7 & $-0.12 \pm 1.2$ & 16 & 90 \\
\hline
\end{tabular}

Abbreviation: OSCC, oral squamous cell carcinoma.

${ }^{a}$ 13-gene DNA methylation analysis was not possible in one brushing sample collected in the group of healthy donors.

b13-gene DNA methylation analysis was not possible in two brushing samples collected in the group of healthy donors.

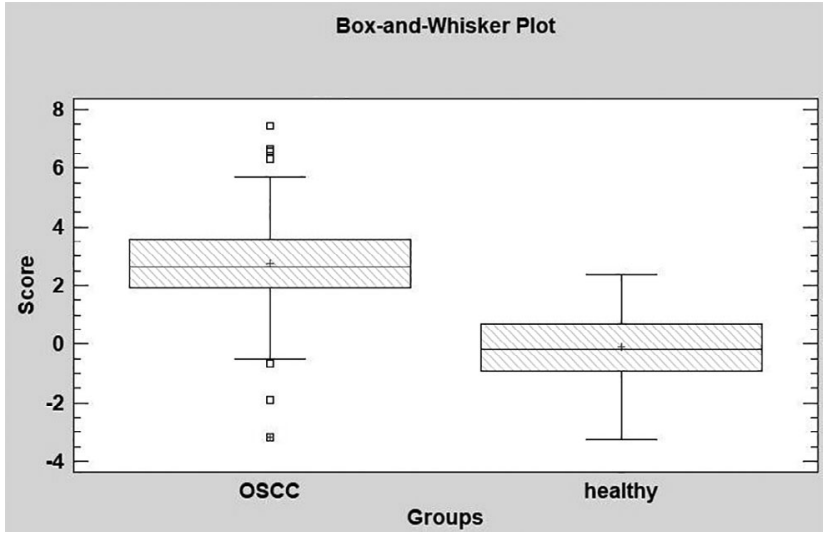

FIG URE 2 Box plot showing the scores calculated using the algorithm showed a significant between-group difference (KruskalWallis test $T=123.1 ; p<0.05)$. The calculated sample scores differed significantly between patients with oral squamous cell carcinoma (OSCC) and healthy volunteers

\section{2 | 13-gene DNA methylation analysis}

A mean score of $2.74 \pm 1.6$ was calculated for brushing specimens collected from patients with OSCC. Scores for 103 of the 110 specimens (93.6\%) exceeded the threshold value and were considered positive. On the other hand, brushing specimens from healthy volunteers had a mean score value of $-0.11 \pm 1.2$. The scores for 16 of the 106 specimens $(15.1 \%)$ from healthy volunteers exceeded

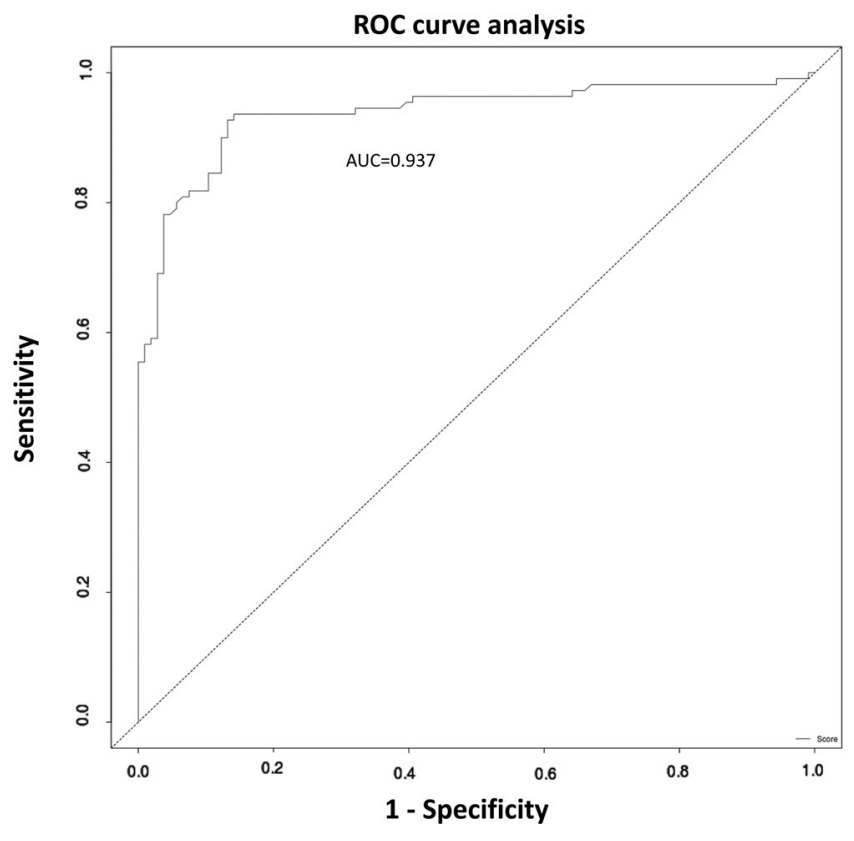

F I G URE 3 Receiver operating characteristics (ROC) analysis showing the ability of the score calculated from the algorithm to discriminate brushing samples acquired from oral squamous cell carcinoma (OSCC) and healthy volunteers. The area under the curve (AUC) was 0.937

the threshold value and were considered positive. None of the healthy volunteers developed OSCC during the study period. Calculated scores and threshold values are summarized in Table 2. 


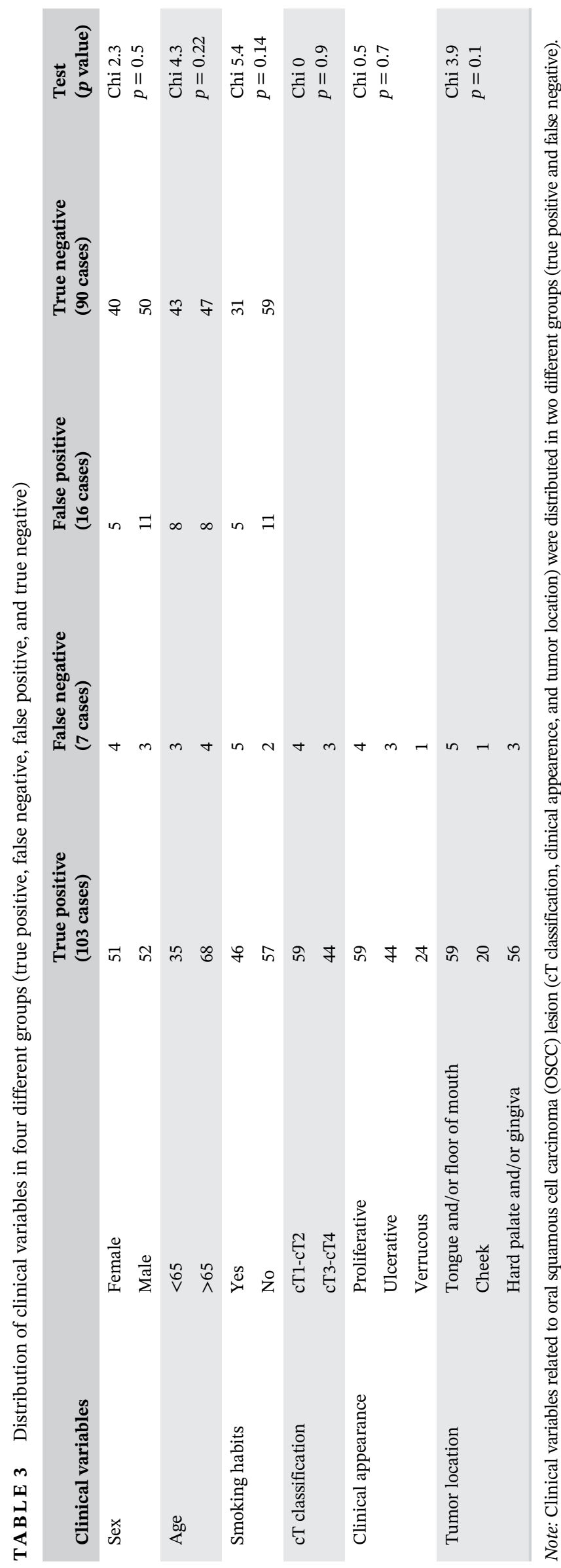

The calculated sample scores differed significantly between patients with OSCC and healthy volunteers, as determined by Fisher's exact test (Chi 134.6, $p<0.000$ ) and Kruskal-Wallis analysis $(T=123.1, p<0.05)$ (Figure 2). Moreover, we found a significant difference between patients with OSCC and healthy volunteers in the CpGs of the 13 genes used to generate the choice algorithm $(p<0.05$; Kruskal-Wallis test; Figure S1, Supporting Information).

The diagnostic value of the 13-gene DNA methylation analysis in oral brushing samples for OSCC detection was assessed using the cutoff value determined in our previous study. ${ }^{13}$ We found that our method provided sensitivity of $93.6 \%$ (CI 87.8-99.5), specificity of $84.9 \%$ (CI 76.2-93.6), PPV of $86.6 \%$ (CI 78.7-94.4), NPV of $92.8 \%$ (CI 86.2-99.4), and accuracy of 89.4\%. Furthermore, ROC curve analysis using the calculated sample scores provided an AUC of 0.937 (Figure 3). ROC analyses were also performed for each $\mathrm{CpG}$ of the 13 genes. The results of these analyses are summarized in Figure S2 and Table S1.

The 216 specimens used in this study were classified into four groups based on the quantitative 13-gene DNA methylation analysis results: true positive cases (TP: patients with OSCC detected as positive; $n=103$ ), false negative cases (FN: patients with OSCC detected as negative; $n=7$ ), false positive cases (FP: healthy volunteers detected as positive; $n=16$ ), and true negative cases (TN: healthy volunteers detected as negative; $n=90$ ). None of the patient variables analyzed in this study (cT classification, tumor location, and clinical appearance for OSCC group and age, sex, and smoking habits for both groups) had a significant effect on the score calculated in these four different groups (Table 3). In particular, the number of $7 \mathrm{FN}$ cases resulted equally distributed on the basis of cT classification (3cT1, 1 cT2, and 3cT4). Heatmap clustering provided two main clusters; the right cluster included $86 \mathrm{TN}$, all $7 \mathrm{FN}, 10 \mathrm{FP}$, and $10 \mathrm{TP}$ samples; the left cluster included $93 \mathrm{TP}, 4 \mathrm{TN}$, and $6 \mathrm{FP}$ samples. The heatmap and ClustVis results are shown in Figures S3 and S4, respectively.

\section{4 | DISCUSSION}

This study is the first multicenter oral oncology study to investigate the diagnostic value of a minimally invasive method for early detection of oral cancer based on genetic and epigenetic analyses. Several studies suggested the potential use of single biomarkers or a panel of biomarkers for early detection of OSCC starting from noninvasive or minimally invasive sampling methods. ${ }^{24-30}$ In particular, different authors recently proposed the 
analysis of DNA methylation status of several genes starting from saliva or oral brushing specimens as promising biomarker. ${ }^{31-38}$ The majority of these authors proposed a study model that included patients with OSCC and non-OSCC normal controls to explore the diagnostic value of potentially OSCC biomarker. However so far, none of these biomarkers has been implemented in clinical practice. In a previous study, we reported the development of a novel assay for early detection of OSCC and HGSIL from oral brushing specimens using bisulfite next-generation sequencing. ${ }^{13}$ Using linear discriminant analysis, we calculated for each sample a score weighted by the best CpGs of a panel of 13 genes. Combination of epigenetic profiles of several genes rather than a single gene as useful source of information in the complex OSCC landscape is in agreement with emerging published literature. Different authors proposed the use a panel of several genes with an aberrant methylation status in $\mathrm{OSCC}^{31,34,35,39}$ rather than a single or few genes $^{32,36-38}$ for diagnostic and prognostic purposes. The ability of our method to discriminate patients with oral cancer from healthy individuals was very high, with an AUC of 0.981. Impressively, 28 of 29 OSCC (96.6\%) and 6 of 6 HGSIL (100\%) specimens exceeded the threshold value, whereas none of the 65 specimens from healthy donors exceeded the threshold.

This performance was achieved by a bisulfite NGS approach, which gives several advantages: a small amount of DNA obtaining from a minimally or noninvasive sampling procedure is necessary for analysis of epigenetic alterations, different genes, and their CpGs usually mapped at the promoter level; quantitative data are available calculating the number of reads with associated unconverted $\mathrm{C}$ in each $\mathrm{CpG}$ position; the pattern of each epiallele in cis may be defined interpreting its homogeneous or heterogeneous behavior; as reported previously by Bock et al. who compared the most promising assays for measuring DNA methylation in clinical diagnostics, best performances were obtained using amplicon bisulfite sequencing with high accuracy and robustness. ${ }^{40}$ (Assigning at least 1000-2000 reads per single region of interest, up to 96 different specimens can be loaded per single run using a micro flow-cell Illumina; moreover, since the library preparation is based on a twostep PCR approach, the total cost of the assay is very limited if compared to other library preparation protocols (see details in Section 2).) In this study, we validated the sensitivity and specificity of the method in an extensive collection of 220 brushing specimens collected in 11 oral medicine units from different parts of Italy. To the best of our knowledge, this is the first multicenter study demonstrating the diagnostic value of a minimally invasive method, which could provide early detection of oral cancer based on genetic and epigenetic analyses.

Our data confirmed the feasibility of the newly developed method. Indeed, in our previous study oral brushing cell collection was always performed by the same operator in a single oral medicine center, whereas in this study 11 different operators collected, stored, and mailed in blindness oral brushing specimens from different oral medicine centers across Italy. Brushing specimens contained sufficient DNA for quantitative DNA methylation analysis, except in 4 of 220 samples in which DNA amplification failed. ROC curve analysis based on the quantitative scores confirmed the high accuracy of the method, with an AUC of 0.937. In particular, 93.6\% (103/110) of the brushing specimens collected from patients with oral neoplastic lesions were identified in blindness as positive, and $84.9 \%(90 / 106)$ of the samples from healthy individuals were identified as negative. No clinical or differences regarding patient variables (age, sex, and smoking habits) and tumor variables (cT classification, tumor location, and clinical appearance) were observed between OSCC specimens with positive and negative scores. Moreover, no significant differences in age, sex, or smoking habits were observed between TN and FP specimens.

While a low number of FPs would minimize unnecessary examinations and anxiety, the development of diagnostic tests with minimal numbers of FN findings is of high clinical importance. ${ }^{8}$ In general, sensitivity and specificity values of at least $80 \%$ are required. It is worth noting that four of seven false-negative samples were consecutive specimens acquired from a single center (center 2). Therefore, the low diagnostic performance of our method in these particular samples may be due to incorrect sampling, leading to the acquisition of insufficient numbers of cancer cells from the lesion. Alternatively, incorrect storage or problems in the mailing procedure may have occurred, as four FP samples were also obtained from the same center. Nevertheless, we cannot exclude the possibility that a small subgroup of OSCCs may show different methylation patterns. Considering that this was a multicenter study, with an extensive number of samples and a wider range of participants, the sensitivity of the method is extremely promising.

No diagnostic tests have been proven to be costeffective for detecting oral lesions in the general population thus far, ${ }^{11}$ as the prevalence of oral cancer in the general population is low. ${ }^{1}$ The fact that our method provides high sensitivity (93.6\%) and NPV (92.7\%) suggests its clinical usefulness as a first-line screening tool in oral cancer detection in the general population, although it should not be ignored the presence of $16 \%$ FP findings reported herein. A strict follow-up of the latter is ongoing 
to verify if they are really FP cases or if they are at increasing risk to develop a lesion later on.

To reduce the number of FP cases and to improve the cost-effectiveness of our procedure, further investigations will be performed to identify local or systemic variables in healthy volunteers (i.e., local benign chronic or acute inflammations, use for systemic drugs) not included in this study design and responsible of an altered methylation profile of one or different genes of our 13-gene panel.

Further investigations will be also performed to evaluate the role of our procedure not only in presence of a suspected neoplastic lesion but also as a surveillance tool for high-risk OSCC groups of patients (i.e., patients with potentially malignant disorders and/or patients surgically treated for OSCC). A study design with brushing cell collection at different moments during the follow-up period of these patients will evaluate the potential role of our procedure as an indicator of disease before the appearance of clinical signs of oral cancer.

\section{5 | CONCLUSION}

Herein, we reported the first multicenter study investigating the feasibility and diagnostic value of a minimally invasive method for the detection of oral cancer based on genetic and epigenetic analyses. Our findings confirmed the high accuracy of the test in an extensive collection of clinical samples. Although the method exhibited high sensitivity, the specificity was slightly lower than expected based on data from our previous study. These findings suggest that this easy-to-perform method could be proposed not in replacement of oral biopsy for definitive diagnosis but as a reliable measure of oral cancer risk, and should be provided to high-risk patients by primary health care providers, dentists, oral medicine specialists, and oral/maxillofacial surgeons.

\section{ACKNOWLEDGMENTS}

This study was supported by an academic grant (Proof of Concept - PoC 2019 assigned to Luca Morandi) from the University of Bologna. The authors thank Roberto Rossi (DDS of Department of Biomedical and Neuromotor Sciences, Section of Oral Sciences, University of Bologna) for support in sample collection and management. We would like to thank all patients and healthy volunteers who participated in the study. The English in this document has been checked by at least two professional editors, both native speakers of English. For a certificate, please see http://www. textcheck.com/certificate/P1UBR7.

\section{CONFLICT OF INTEREST}

As a possible conflict of interest, Luca Morandi, Davide B. Gissi, and Achille Tarsitano submitted a patent (the applicant is the University of Bologna) in November 2016 to the National Institute of Industrial Property; however, we believe that this is a natural step of translational research (bench-to-bedside) and guarantee that the scientific results are true. The remaining authors declare that they have no conflict of interests.

\section{DATA AVAILABILITY STATEMENT}

Data available on request from the authors.

\section{ORCID}

Davide B. Gissi (1D https://orcid.org/0000-0002-0195-7694

Roberto De Luca (1D) https://orcid.org/0000-0002-7980-9798

\section{REFERENCES}

1. Bray F, Ferlay J, Soerjomataram I, Siegel RL, Torre LA, Jemal A. Global cancer statistics 2018: GLOBOCAN estimates of incidence and mortality worldwide for 36 cancers in 185 countries. CA Cancer J Clin. 2018;68(6):394-424.

2. Ng JH, Iyer NG, Tan M-H, Edgren G. Changing epidemiology of oral squamous cell carcinoma of the tongue: a global study. Head Neck. 2017;39(2):297-304.

3. Braakhuis BJM, Tabor MP, Leemans CR, van der Waal I, Snow GB, Brakenhoff RH. Second primary tumors and field cancerization in oral and oropharyngeal cancer: molecular techniques provide new insights and definitions. Head Neck. 2002;24(2):198-206.

4. Lippman SM, Hong WK. Second malignant tumors in head and neck squamous cell carcinoma: the overshadowing threat for patients with early-stage disease. Int J Radiat Oncol Biol Phys. 1989;17(3):691-694.

5. National Cancer Institute. Surveillance, epidemiology, and end results program: oral cavity an pharynx cancer. https://seer. cancer.gov/statfacts/html/oralcav.html.

6. Brenner $H$. Long-term survival rates of cancer patients achieved by the end of the 20th century: a period analysis. Lancet. 2002;360(9340):1131-1135.

7. Thomson PJ. Field change and oral cancer: new evidence for widespread carcinogenesis? Int J Oral Maxillofac Surg. 2002;31 (3):262-266.

8. Brocklehurst PR, Speight PM. Screening for mouth cancer: the pros and cons of a national programme. Br Dent J. 2018;225(9): 815-819.

9. Giovannacci I, Vescovi P, Manfredi M, Meleti M. Non-invasive visual tools for diagnosis of oral cancer and dysplasia: a systematic review. Med Oral Patol Oral Cir Bucal. 2016;21(3):e305e315.

10. Morikawa T, Shibahara T, Nomura T, Katakura A, Takano M. Non-invasive early detection of oral cancers using fluorescence visualization with optical instruments. Cancers. 2020;12(10): 2771.

11. Brocklehurst $\mathrm{P}$, Kujan O, Glenny A-M, et al. Screening programmes for the early detection and prevention of oral cancer. Cochrane Database Syst Rev. 2010;(11):CD004150. 
12. Kaur J, Jacobs R, Huang Y, Salvo N, Politis C. Salivary biomarkers for oral cancer and pre-cancer screening: a review. Clin Oral Investig. 2018;22(2):633-640.

13. Morandi L, Gissi D, Tarsitano A, et al. CpG location and methylation level are crucial factors for the early detection of oral squamous cell carcinoma in brushing samples using bisulfite sequencing of a 13-gene panel. Clin Epigenetics. 2017;9:85.

14. Gissi DB, Tarsitano A, Gabusi A, et al. 13-gene DNA methylation analysis from oral brushing: a promising non invasive tool in the follow-up of oral cancer patients. J Clin Med. 2019;8(12):2107.

15. Morandi L, Gissi D, Tarsitano A, et al. DNA methylation analysis by bisulfite next-generation sequencing for early detection of oral squamous cell carcinoma and high-grade squamous intraepithelial lesion from oral brushing. J Craniomaxillofac Surg. 2015;43(8):1494-1500.

16. Gissi DB, Morandi L, Gabusi A, et al. A noninvasive test for MicroRNA expression in oral squamous cell carcinoma. Int $J$ Mol Sci. 2018;19(6):1789.

17. Morandi L, Righi A, Maletta F, et al. Somatic mutation profiling of hobnail variant of papillary thyroid carcinoma. Endocr Relat Cancer. 2017;24(2):107-117.

18. Afgan E, Baker D, Batut B, et al. The Galaxy platform for accessible, reproducible and collaborative biomedical analyses: 2018 update. Nucleic Acids Res. 2018;46(W1):W537-W544.

19. Hu K, Ting AH, Li J. BSPAT: a fast online tool for DNA methylation co-occurrence pattern analysis based on high-throughput bisulfite sequencing data. BMC Bioinformatics. 2015;16:220.

20. Krainer J, Weinhäusel A, Hanak K, et al. EPIC-TABSAT: analysis tool for targeted bisulfite sequencing experiments and array-based methylation studies. Nucleic Acids Res. 2019;47 (W1):W166-W170.

21. Gruntman E, Qi Y, Slotkin RK, Roeder T, Martienssen RA, Sachidanandam R. Kismeth: analyzer of plant methylation states through bisulfite sequencing. BMC Bioinformatics. 2008;9:371.

22. Goksuluk D, Korkmaz S, Zararsiz G, Karaagaoglu AE. easyROC: an interactive web-tool for ROC curve analysis using R language environment. $R$ J. 2016;8(2):213-230.

23. Metsalu T, Vilo J. ClustVis: a web tool for visualizing clustering of multivariate data using principal component analysis and heatmap. Nucleic Acids Res. 2015;43(W1):W566-W570.

24. Tang KD, Kenny L, Perry C, Frazer I, Punyadeera C. The overexpression of salivary cytokeratins as potential diagnostic biomarkers in head and neck squamous cell carcinomas. Oncotarget. 2017;8(42):72272-72280.

25. Lim Y, Fukuma N, Totsika M, Kenny L, Morrison M, Punyadeera $\mathrm{C}$. The performance of an oral microbiome biomarker panel in predicting pral cavity and oropharyngeal cancers. Front Cell Infect Microbiol. 2018;8:267.

26. Franzmann EJ, Reategui EP, Pereira LHM, et al. Salivary protein and solCD44 levels as a potential screening tool for early detection of head and neck squamous cell carcinoma. Head Neck. 2012;34(5):687-695.

27. Wang Q, Gao P, Wang X, Duan Y. Investigation and identification of potential biomarkers in human saliva for the early diagnosis of oral squamous cell carcinoma. Clin Chim Acta. 2014;427:79-85.

28. Peisker A, Raschke G-F, Fahmy M-D, et al. Salivary MMP-9 in the detection of oral squamous cell carcinoma. Med Oral Patol Oral Cir Bucal. 2017;22(3):e270-e275.
29. Pereira LHM, Reis IM, Reategui EP, et al. Risk stratification system for oral cancer screening. Cancer Prev Res (Phila). 2016; 9(6):445-455.

30. Shi L, Wang Y, Li C, Liu W. Current evidence on DNA aneuploidy cytology in noninvasive detection of oral cancer. Oral Oncol. 2020;101:104367.

31. Nagata S, Hamada T, Yamada N, et al. Aberrant DNA methylation of tumor-related genes in oral rinse: a noninvasive method for detection of oral squamous cell carcinoma. Cancer. 2012; 118(17):4298-4308.

32. Huang Y-K, Peng B-Y, Wu C-Y, Su C-T, Wang H-C, Lai H-C. DNA methylation of PAX1 as a biomarker for oral squamous cell carcinoma. Clin Oral Investig. 2014;18(3):801-808.

33. Puttipanyalears C, Arayataweegool A, Chalertpet K, et al. TRH site-specific methylation in oral and oropharyngeal squamous cell carcinoma. BMC Cancer. 2018;18(1):786.

34. Arantes LMRB, de Carvalho AC, Melendez ME, et al. Validation of methylation markers for diagnosis of oral cavity cancer. Eur J Cancer. 2015;51(5):632-641.

35. Liyanage C, Wathupola A, Muraleetharan S, Perera K, Punyadeera C, Udagama P. Promoter hypermethylation of tumor-suppressor genes p16INK4a, RASSF1A, TIMP3, and $P C Q A P / M E D 15$ in salivary DNA as a quadruple biomarker panel for early detection of oral and oropharyngeal cancers. Biomolecules. 2019;9(4):148.

36. Cheng S-J, Chang C-F, Ko H-H, et al. Hypermethylated ZNF582 and $P A X 1$ genes in mouth rinse samples as biomarkers for oral dysplasia and oral cancer detection. Head Neck. 2018;40(2):355-368.

37. Kordi-Tamandani DM, Moazeni-Roodi A-K, Rigi-Ladiz M-A, Hashemi M, Birjandian E, Torkamanzehi A. Promoter hypermethylation and expression profile of MGMT and CDH1 genes in oral cavity cancer. Arch Oral Biol. 2010;55(10):809-814.

38. Demokan S, Chang X, Chuang A, et al. KIF1A and EDNRB are differentially methylated in primary HNSCC and salivary rinses. Int J Cancer. 2010;127(10):2351-2359.

39. Guerrero-Preston R, Michailidi C, Marchionni L, et al. Key tumor suppressor genes inactivated by "greater promoter" methylation and somatic mutations in head and neck cancer. Epigenetics. 2014;9(7):1031-1046.

40. Bock C, Tomazou EM, Brinkman AB, et al. Quantitative comparison of genome-wide DNA methylation mapping technologies. Nat Biotechnol. 2010;28(10):1106-1114.

\section{SUPPORTING INFORMATION}

Additional supporting information may be found online in the Supporting Information section at the end of this article.

How to cite this article: Gissi DB, Morandi L, Colella G, et al. Clinical validation of 13-gene DNA methylation analysis in oral brushing samples for detection of oral carcinoma: An Italian multicenter study. Head \& Neck. 2021;1-11. https://doi.org/10. 1002/hed.26624 